Tropical Journal of Pharmaceutical Research March 2017; 16 (3): 641-647

ISSN: $1596-5996$ (print); 1596-9827 (electronic)

(C) Pharmacotherapy Group, Faculty of Pharmacy, University of Benin, Benin City, 300001 Nigeria.

All rights reserved.

Available online at http://www.tjpr.org

Original Research Article

http://dx.doi.org/10.4314/tjpr.v16i3.20

\title{
Evaluation of MicroRNA 125b as a potential biomarker for postmenopausal osteoporosis
}

\author{
Hua Chen ${ }^{1}$, Honghui Jiang ${ }^{2}$, Dan Can ${ }^{2}$, Hao Xu ${ }^{3}$, Keliang Zhang ${ }^{3}$, Song Guo ${ }^{3 \star}$ \\ ${ }^{1}$ Department of Orthopaedic Surgery, Hubei Provincial Hospital of TCM, Wuhan, 430061, ${ }^{2}$ Department of Orthopaedic Surgery, \\ The Central Hospital of Wuhan, Tongji Medical College Huazhong University of Science \& Technology, Wuhan, 430030, \\ ${ }^{3}$ Department of Orthopaedic Surgery, Puai Hospital of Wuhan, Tongji Medical College Huazhong University of Science \& \\ Technology, Wuhan, 4300033, China
}

*For correspondence: Email: songguo134@hotmail.com; Tel: 0086-27-68831300

Received: 22 November 2016

Revised accepted: 24 February 2017

\begin{abstract}
Purpose: To identify significant dysregulated miRNAs in postmenopausal osteoporosis in Chinese women and to test whether any of these miRNAs have diagnostic potential as circulatory biomarkers for postmenopausal osteoporosis.

Methods: Thirty osteoporotic patients and 30 non-osteoporotic healthy individuals were recruited, and blood and bone tissue samples were collected from them. miRNA expression profiling and quantitative real-time polymerase chain reaction ( $q R T-P C R)$ were used to identify and substantiate dysregulated miRNAs in blood sera and bone tissue from osteoporotic patients. Receiver operating characteristic curve (ROC) analysis was carried out to assess the diagnostic potential of significantly dysregulated miRNAs.

Results: Based on profiling and qRT-PCR, miR-125b, miR-30 and miR-5914 were significantly upregulated in the blood sera and bone tissues of patients with postmenopausal osteoporosis. In all the experiments carried out, miR-125b showed the highest levels of upregulation both in the blood sera and bone tissue compared to other upregulated miRNAs in osteoporotic patients. ROC analysis indicate that the AUC of miR-125b was the highest amongst the upregulated miRNAs.

Conclusion: miR-125b is the highest significantly upregulated miRNA in postmenopausal osteoporosis. Furthermore, circulating miR-125b has the potential of a non-invasive biomarker for postmenopausal osteoporosis.
\end{abstract}

Keywords: Postmenopausal osteoporosis, Profiling, Up-regulation, miR-125b, Biomarker

Tropical Journal of Pharmaceutical Research is indexed by Science Citation Index (SciSearch), Scopus, International Pharmaceutical Abstract, Chemical Abstracts, Embase, Index Copernicus, EBSCO, African Index Medicus, JournalSeek, Journal Citation Reports/Science Edition, Directory of Open Access Journals (DOAJ), African Journal Online, Bioline International, Open-J-Gate and Pharmacy Abstracts

\section{INTRODUCTION}

Osteoporosis is a disease wherein the bones become porous and the bone mineral density decreases thereby increasing the chances of broken bones. Numerous studies have shown that fragility of the bones is due to imbalances between bone resorption, responsible by osteoclasts and bone deposition, responsible by osteoblasts $[1,2]$. Statistical studies have shown that osteoporosis is more prevalent in women than in men with about $8 \%$ men and $38 \%$ women being affected in the developed world [3]. Postmenopausal women constitute a high-risk group because of a direct correlation between dwindling estrogen levels and development of osteoporosis, ultimately resulting in the bones to become brittle and break [4].

MicroRNAs are small non-coding RNAs of 22-25 nucleotides that have been shown to play indispensable regulatory roles in gene 
expression in plant and animal kingdoms [5]. Since miRNAs are centerpieces of gene regulatory networks, varying expression levels of such regulatory miRNAs have been often associated with various types of cancers, cardiovascular diseases, neurodevelopmental diseases, autoimmune diseases and skeletal muscle diseases [6]. Accumulating evidence in the past few years have suggested that miRNAs play important regulatory roles in bone metabolism. Sugatani and Hruska have shown that miR-223 is expressed in mouse osteoclasts and plays a pivotal role in osteoclast differentiation [7]. Li et al have identified a previously unknown miRNA, miR-2861, that represses histone deacetylase 5 (HDAC5) expression thereby promoting osteoblast differentiation [8]. Further, they showed that miR2861 contributes to osteoporosis through its effect on osteoblasts. More recently, Xiao et al demonstrated the role of microRNA, miR-129-5p in osteoblast differentiation and bone homeostasis [9].

Accumulating evidence in the last decade has suggested the diagnostic and prognostic potential of miRNAs in a variety of diseases, notably various types of cancer [10]. A most recent study identified a range of dysregulated miRNAs in osteoporosis related pathways and suggests miR-194-5p as a viable biomarker for postmenopausal osteoporosis [11]. Similarly, miR133a and miR422a have been identified as potential biomarkers for postmenopausal osteoporosis $[12,13]$. The present study aims to expand the repertoire of dysregulated miRNAs in postmenopausal osteoporosis and identify other potential miRNA biomarkers for early detection of postmenopausal osteoporosis.

\section{METHODS}

\section{Patient samples and characteristics}

The institutional review board and the ethical committee of Puai Hospital of Wuhan, China reviewed and approved the study (approval no. YX3254576) and written consent was obtained from every participant involved in this study. All procedures performed in this study involving human participants were in accordance with the ethical standards of the institutional and/or national research committee and with the 1964 Helsinki declaration and its later amendments or comparable ethical standards [21].

Thirty postmenopausal Chinese women with osteoporosis constituted the osteoporotic or the patient group. All the patients were past menopause in the age group of $59-80$ years. The recruited patients carried hip fractures (in femoral neck, trochanter, and intertrochanteric regions) that required surgical intervention. This study did not recruit any patients having other medical issues such as malignancy or cancers, inflammation, diabetes, cardiovascular and metabolic disorders. Blood and bone tissue were collected from this postmenopausal osteoporotic patient pool and carefully harvested and stored for further analysis. The patient characteristics are summarized in Table 1.

\section{Isolation of RNA from serum and bone}

Five milliliters of blood was obtained from each patient and the blood was allowed to clot. Following clotting, the samples were centrifuged at $1500 \mathrm{~g}$ and the supernatant containing serum was isolated and stored. MiRNAs from the blood sera samples were isolated using Qiagen miRNeasy Serum/Plasma Kit (Qiagen, Hilden, Germany) according to the protocol provided in the handbook with minor modifications. The final RNA mixture was eluted in $20 \mu \mathrm{L}$ of Rnase free water and stored at $-80^{\circ} \mathrm{C}$. miRNA from bone tissue was extracted using miRCURRY RNA Isolation Kit - Tissue (Exiqon Life Sciences, Copenhagen, Denmark) according to the instructions provided in the manual. The final RNA mixture was eluted in $20 \mu \mathrm{L}$ of Rnase free water and stored at $-80^{\circ} \mathrm{C}$.

Table 1: Characteristics of subjects involved in this study

\begin{tabular}{lcc}
\hline Characteristics & $\begin{array}{c}\text { Osteoporotic patients } \\
(\mathrm{N}=30)\end{array}$ & $\begin{array}{c}\text { Non-osteoporotic patients } \\
\mathrm{N}=30\end{array}$ \\
\hline Sex & 30 females, 0 males & 30 females, 0 males \\
Age range (years) & $59-80$ & $62-75$ \\
Height range $(\mathrm{cm})$ & $155-178$ & $153-181$ \\
Weight $(\mathrm{kg})$ & $62-96$ & $58-103$ \\
Body mass index,$\pm \mathrm{SD}$ & $23.8 \pm 5.7$ & $25.2 \pm 3.8$ \\
Bone density $(\mathrm{mg} / \mathrm{dl}), \pm \mathrm{SD}$ & $83.6 \pm 19.9$ & $197 \pm 35.6$ \\
Other medical condition & None & None \\
\hline
\end{tabular}




\section{Quantitative real time polymerase chain reaction}

The dysregulated miRNAs were further validated by qRT-PCR and the RNA eluates were reverse transcribed to cDNA using TaqMan MicroRNA Reverse Transcription Kit (ThermoScientific, Waltham, USA) according to the instructions provided in the manual. Briefly, $10 \mathrm{ng}$ of total RNA in reaction volume of $15 \mu \mathrm{L}$ of reverse transcription reactions were prepared in $1.5 \mu \mathrm{L}$ of 10X RT buffer, $0.15 \mu \mathrm{L}$ of $100 \mathrm{mM}$ dNTPs, $0.2 \mu \mathrm{L}$ of 20 units/ $\mu \mathrm{L}$ RNase-inhibitor, $1 \mu \mathrm{L}$ of each of microRNA primers (100 mM), $1 \mu \mathrm{L}$ of MultiScribe Reverse Transcriptase (50 units/ $\mu \mathrm{L}$ ), and 10.15 $\mu \mathrm{L}$ of DEPC treated water. The RT protocol was as follows: $18{ }^{\circ} \mathrm{C}$ for $30 \mathrm{~min}, 42{ }^{\circ} \mathrm{C}$ for $30 \mathrm{~min}$ and $90{ }^{\circ} \mathrm{C}$ for $5 \mathrm{~min}$. Quantitative real-time PCR was performed on a $7900 \mathrm{HT}$ Fast Real-Time system (Applied Biosystems, California, USA). The reaction conditions were: $95^{\circ} \mathrm{C}$ for $10 \mathrm{~min}$ and 35 cycles of $95^{\circ} \mathrm{C}$ for $20 \mathrm{~s}$ and $58^{\circ} \mathrm{C}$ for 1 min. U6 RNA was used as endogenous control for all the reactions. The relative quantity of each miRNA was determined by $2^{-\triangle \triangle C T}$ method, where CT indicates cycle threshold, $\triangle \mathrm{CT}=(\mathrm{CT}$ target miRNA - CT endogenous control U6 RNA) and $\triangle \triangle \mathrm{CT}=$ ( $\triangle \mathrm{CT}-$ average $\Delta \mathrm{CT}$ of all the samples). Sequences of primers used are listed in Table 2.

\section{Statistical analysis}

The data analyzed are expressed as the mean \pm standard deviation (SD). For analysis and determination of significance, two-tailed MannWhitney $U$ test or Student's $t$-test was used. GraphPad Prism software (Version 6) was used to conduct statistical analysis. ROC curve analysis was used to determine the diagnostic potential of specific upregulated miRNAs. In all cases when interpreting the data, a value of $p<$ 0.05 was considered statistically significant.

\section{RESULTS}

\section{Dysregulated miRNAs in the sera of postmenopausal osteoporosis patients}

A comprehensive miRNA expression profiling was carried out from a pool of 30 RNA samples isolated from patients with postmenopausal osteoporosis and from 30 healthy nonosteoporotic Chinese women of the same age group, which served as control (Table 1). A total of 95 miRNAs could be detected in the array, and of those 95 detected miRNAs, 5 miRNAs (miR125b, miR-30, miR-4665-3p, miR-5914 and miR96) showed significant upregulation in the sera of postmenopausal osteoporotic patients compared to the healthy non-osteoporotic controls (Figure 1). Amongst the five significantly upregulated miRNAs in postmenopausal osteoporotic patients, miR-125b was the most upregulated with a 12.67-fold change, followed by miR-4665$3 p$ with a 7.34-fold change, miR-96 with a 3.17fold change, miR-30 and miR-5914 both with a 1.5 -fold change compared to the samples from non-osteoporotic healthy individuals.

\section{miR-125b is significantly upregulated in serum of postmenopausal osteoporotic patients}

Using q-RTPCR, we validated whether any of the "best" dysregulated miRNAs, obtained by profiling, can be used as cues to distinguish serum samples of postmenopausal osteoporotic patients from that of non-osteoporotic healthy individuals. Since the data from qRT-PCR is more robust due to its much lower false positive rate than compared with profiling experiments, we resorted to the same to conclusively establish the plausibility of any such miRNA acting as a biomarker for postmenopausal osteoporosis. The qRT-PCR validation set consisted of serum samples from 30 postmenopausal osteoporotic patients and 30 non-osteoporotic healthy individuals. The expression levels of miR-4665$3 p$ and miR-96 were similar between the osteoporotic and non-osteoporotic sample group (Figure 2). However, the levels of miR-125b, miR-30 and miR-5914 were higher in osteoporotic serum samples than the healthy ones (Figure 2). Between the above stated threeupregulated miRNAs, miR-125b was the most significant suggesting that miR-125b has the potential as a biomarker for postmenopausal osteoporosis.

Table 2: Primer sequences used in the study

\begin{tabular}{lll}
\hline Gene & Forward primer (5'-3') & Reverse primer (5'-3') \\
\hline U6 & GCTTCGGCACATATACTAAAATA & CGCTTCACGAATTTGCGTGT \\
miR-30 & GTGCCCAGATCAAGGTCGC & CGACTGCGTGTCGACCAGC \\
miR-96 & GCTATGACGGAATCGCCAG & CATGGTGCTTGTTGCGAGAT \\
miR-125b & GCCGACATGCTAGAAACGCCTC & CCTAGCTGAGGTACACCCAGG \\
miR-5914 & GCGGGCGGATCGAATGATCGCG & GCTAGGATGTCCGGATGGAGG \\
miR-4665-3p & GCGCGCGATCACGGCGTTACTAT & CTACTGGACGGTAAAGCCGG \\
\hline
\end{tabular}




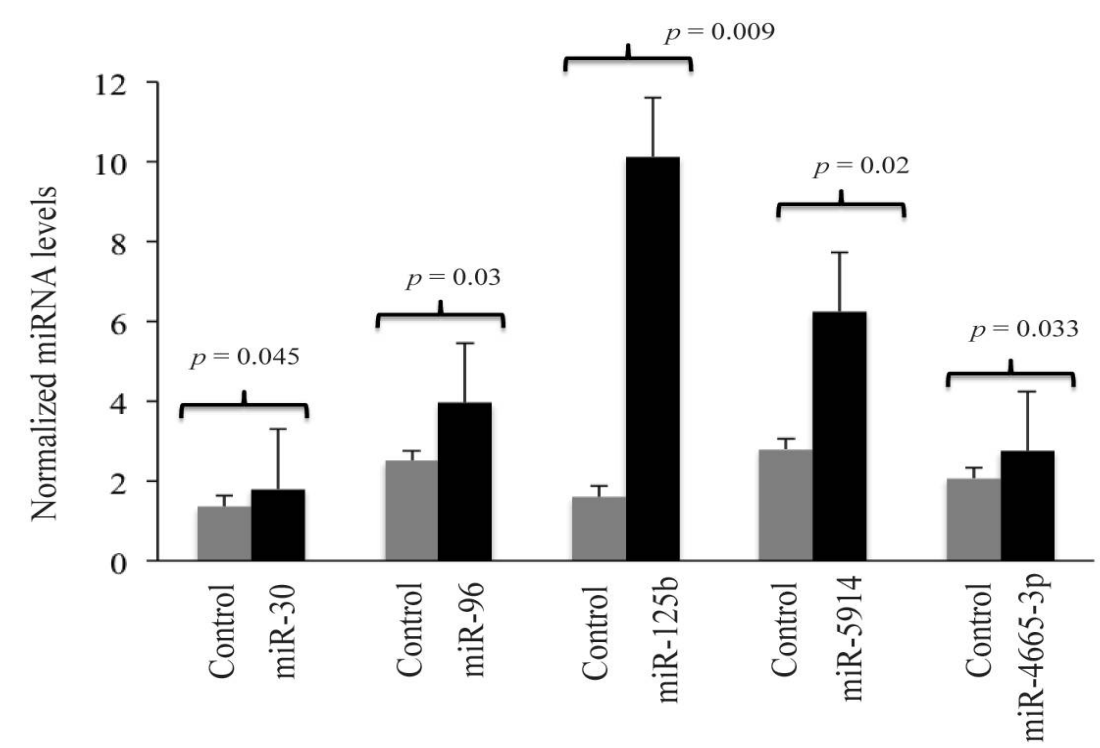

Figure 1: Expression profiling of miRNAs in the sera of postmenopausal osteoporotic patients with respect to healthy non-osteoporotic samples or control samples. It can be seen that all 5 miRNAs are significantly dysregulated, and of them, miR-125b shows maximum upregulation $(p=0.009)$ in the osteoporotic samples followed by miR-5914 $(p=0.02)$, miR-96 $(p=0.03)$, miR-4665-3p $(p=0.033)$ and miR-30 $(p=0.045)$

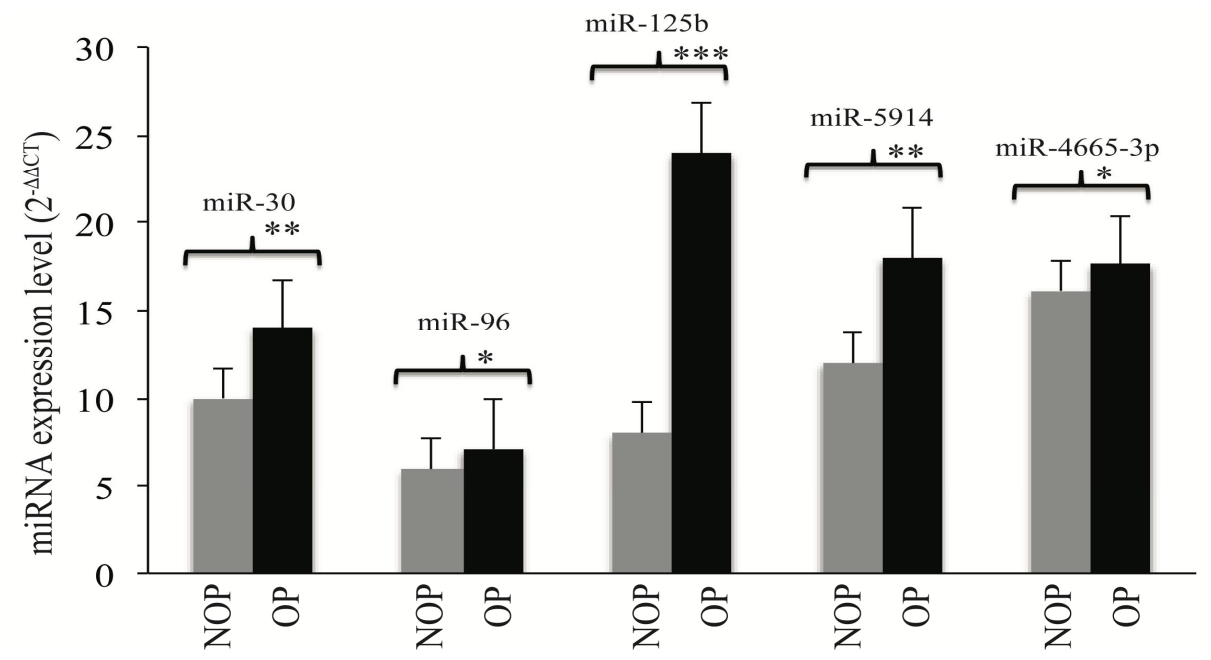

Figure 2: Substantiation of dysregulated miRNAs from profiling experiments by quantitative real time PCR. The miRNA expression levels were calculated by $2^{-\Delta \Lambda C T}$ method. The expression levels of miR-96 and miR-4665-3p were similar between the osteoporotic group (OP) and the non-osteoporotic group (NOP). The expression levels of miR-30, miR-125b and miR5914 were higher in OP group than in NOP group. Amongst the three-upregulated miRNAs, miR-125b showed the highest levels of upregulation followed by miR-5914 and mi-30; * indicates $p<$ $0.05,{ }^{* *}$ indicates $p<0.03$ and ${ }^{* * *}$ indicates $p<0.01$ )

\section{miR-125b is significantly upregulated in the bone tissues of postmenopausal osteoporotic patients}

We proceeded to investigate whether any of the dysregulated miR125b, miR30 and miR-5914 could be detected in the bone tissues of postmenopausal osteoporotic patients. Consistent with the qRT-PCR results, the levels of all the three miRNAs were high compared with the healthy non-osteoporotic bone tissue, signifying upregulation of all three miRNAs (Figure 3). Between the three-upregulated
miRNAs, the levels of miR-125b were significantly higher in the bone tissue of postmenopausal osteoporotic patients (Figure 3). These results are similar to the serum analysis further strengthening the potential of miR-125b as a biomarker for postmenopausal osteoporosis.

\section{Diagnostic potential of miR-125b in postmenopausal osteoporosis}

To assess the diagnostic potential of miR-125b in postmenopausal osteoporosis, a routinely used 
statistical method, receiver operating characteristic (ROC) curve analysis was performed. The ROC analysis was extended to miR-30 and miR-5914 to assess the diagnostic value of all these miRNAs. For each miRNA, the area under the curve (AUC), standard error and confidence interval was calculated (Figure 4). The AUC of miR-125b was calculated to be 0.8944 (95\% confidence interval [Cl] 0.98670.9934 , Standard error 0.00795 ), that of miR-30 was calculated to be 0.7574 (95\% confidence interval [Cl] 0.9153-0.9866, Standard error 0.0312 ), and that of miR-5914 was calculated to be 0.6988 (95\% confidence interval [Cl] 0.9517 0.9864 , Standard error 0.028 ). The AUC of miR-
$125 \mathrm{~b}$ was found to be the highest amongst the miRNAs and clearly suggest that miR $-125 \mathrm{~b}$ has a strong potential diagnostic value for postmenopausal osteoporosis detection.

\section{DISCUSSION}

Osteoporosis has been a growing heath concern in aging women in the developing world, especially in China [14]. Women that past are menopause, in particular, have been susceptible to osteoporosis eventually resulting in fragility and fractures in the bones as elaborated in the introduction section.

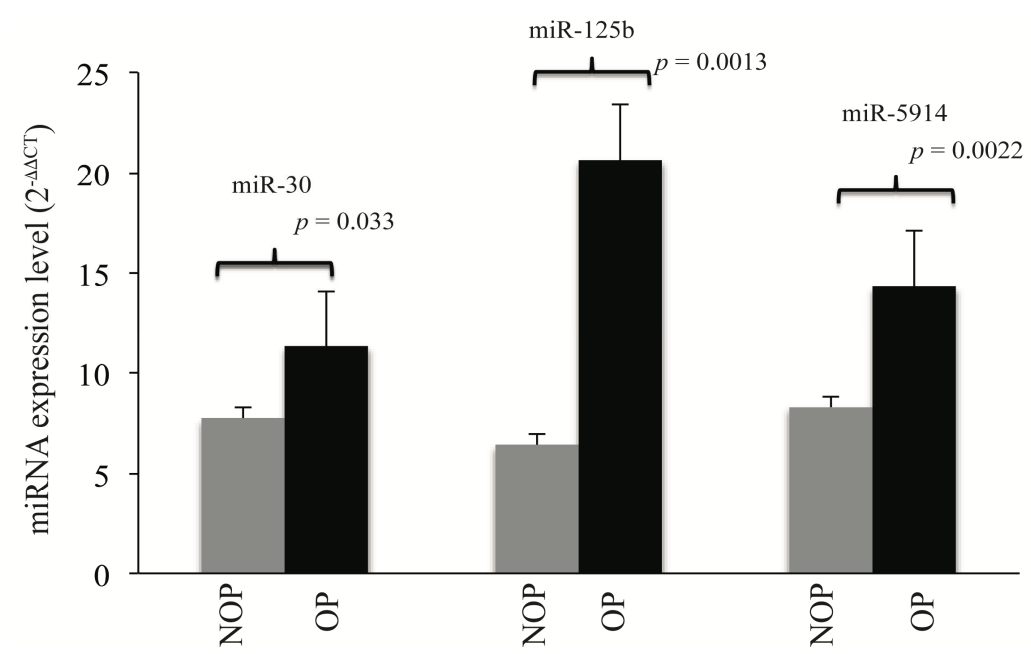

Figure 3: miR-30, miR-125b and miR-5914 were significantly upregulated in the bone tissues of osteoporotic group (OP) compared to non-osteoporotic group (NOP). miR-125b showed highest levels of upregulation $(p=$ $0.0013)$ followed by miR-5914 $(p=0.0022)$ and miR-30 $(p=0.033)$. The miRNA expression levels in the bone tissues from both OP and NOP were calculated by $2^{-\triangle \triangle C T}$ method
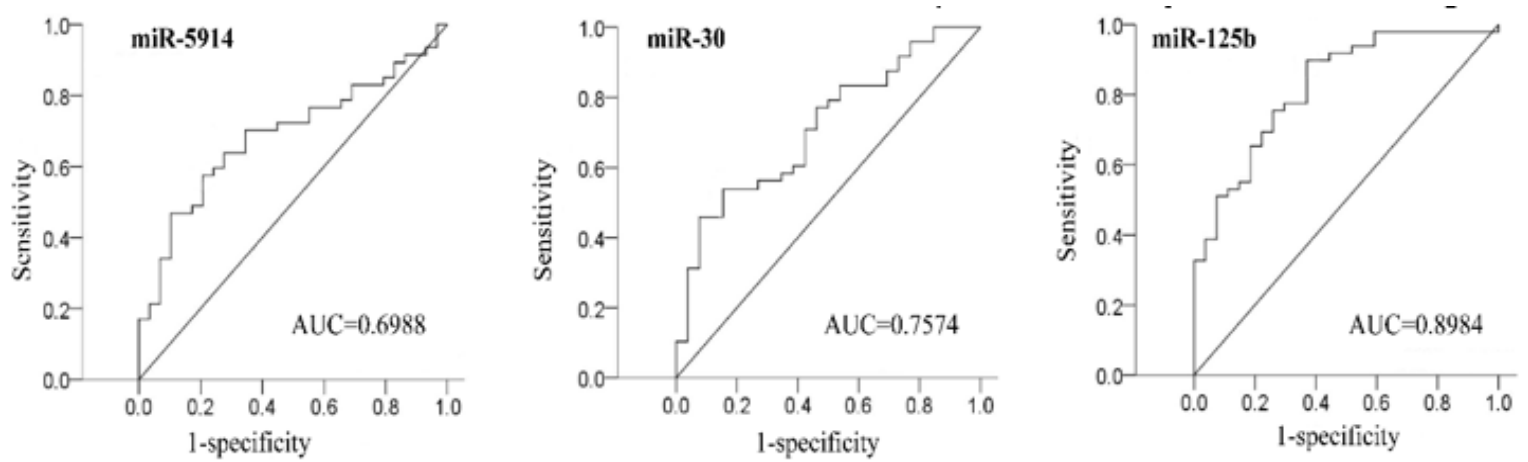

Figure 4: Diagnostic potential of miR-5914, miR-30 and miR-125b for postmenopausal osteoporosis. ROC curves were plotted with sensitivity on the $y$-axis indicating a true positive rate and 1-specificity on the $x$-axis indicating false positive rate. The area under the curve (AUC) for each miRNA was estimated from the ROC curves. The AUC was found to be the best for miR-125b (0.8984) indicating a very good diagnostic potential, followed by miR-30 (0.7574) and miR-5914 (0.6988)

This study has been designed and conducted to identify any potential miRNA that could serve as a biomarker for postmenopausal osteoporosis. By adopting a top-down approach, and using a combination of miRNA profiling followed by robust validation using quantitative real time PCR, this study revealed that miR-125b is significantly upregulated in the blood and bone tissue of postmenopausal osteoporotic patients, having promising diagnostic potential. 
The identification of miRNAs as biomarkers for various disease conditions has been well established. Cumulating literary evidence in the past decade has suggested that miRNAs not only carry a wealth of information for the prognosis and diagnosis of a wide range of diseases but also are also useful in monitoring treatment response [10]. Till date, to the best of our knowledge, there have been only a handful of studies demonstrating the predictive value of specific miRNAs as biomarkers for osteoporosis. In one of such studies, 9 miRNAs were upregulated in the serum, 6 miRNAs in the bone tissue, and 5 miRNAs in both the serum and the bone tissue of osteoporotic patients, suggesting they may be used as biomarkers for diagnostic purposes [15]. Recently, miRNA expression analysis of circulating monocytes has been carried out in vivo and the study suggested miR$133 a$ as a potential biomarker for postmenopausal osteoporosis [12]. The aforestated study was one of its first kinds in identifying miR-133a as a biomarker in circulating monocytes. The same group identified another miRNA in circulating monocytes, miR-422a, as a potential biomarker for postmenopausal osteoporosis [13]. More recently, 5 dysregulated miRNAs in osteosarcoma were identified in microarray analysis and upon further subjection to pathway analysis only miR-194-5p was enriched in osteoporosis related pathways suggesting miR-194-5p as a potential biomarker for postmenopausal osteoporosis [11].

In a quest to identify other potential miRNA biomarkers for postmenopausal osteoporosis, in the current study, serum and bone tissue from 30 postmenopausal osteoporotic Chinese women were investigated for miRNA dysregulation. MiRNA profiling in the serum revealed 5 miRNAs, namely miR-125b, miR-30, miR-4665$3 p$, miR-5914 and miR-96 to be upregulated compared to healthy controls. Interestingly, miR$125 \mathrm{~b}$ showed maximum fold change when compared to other upregulated miRNAs. The major disadvantage of such profiling, owing to inherent technical limitations, is the high false positive rate resulting in erroneous results and interpretation.

To perform a more stringent and robust analysis, qRT-PCR was used to substantiate the upregulated miRNAs. The analysis clearly indicated that the expression levels of miR-125b, miR-30 and miR-5914 were elevated in the serum of osteoporotic patients, with miR-125b showing the highest levels amongst all. This finding gave us initial clues that miR-125b could be a potential biomarker for postmenopausal osteoporosis. Previously, circulating miR-125b has been shown as a potential biomarker for Alzheimer's disease [16], rectal adenocarcinoma [17], Ewing's sarcoma [18], breast [19] and nonsmall cell lung cancer [20]. To the best of our knowledge, there has not been any detailed and conclusive study suggesting the diagnostic potential of miR-125b in postmenopausal osteoporosis.

The next step was to investigate whether any of these upregulated miRNAs were found at elevated levels in the bone tissue. Consistent with the qRT-PCR validation results, all the three miRNAs, miR-125b, miR-30 and miR-5914, were upregulated in the bone tissue of osteoporotic patients with miR-125b showing highest levels of expression. Taken together, this analysis indicates that miR-125b is significantly upregulated, in both sera and bone in postmenopausal osteoporotic patients. Additionally, the diagnostic potential of the upregulated miRNA pool was probed by ROC curve analysis that revealed miR-125b as the most promising candidate as a potential biomarker.

\section{CONCLUSION}

Altogether, the findings of the present study clearly demonstrate that miR-125b is significantly upregulated in postmenopausal osteoporosis and has the potential to serve as suitable and lowcost serum-based biomarker for postmenopausal osteoporosis.

\section{DECLARATIONS}

\section{Acknowledgement}

The authors are indebted to Hubei Provincial Hospital, Central Hospital of Wuhan and Puai Hospital of Wuhan for unrestricted access to all their facilities and machines.

\section{Conflict of Interest}

No conflict of interest associated with this work.

\section{Contribution of Authors}

The authors declare that this work was done by the authors named in this article and all liabilities pertaining to claims relating to the content of this article will be borne by them.

\section{Open Access}

This is an Open Access article that uses a funding model which does not charge readers or 
their institutions for access and distributed under the terms of the Creative Commons Attribution License (http://creativecommons.org/licenses/by 14.0) and the Budapest Open Access Initiative (http://www.budapestopenaccessinitiative.org/rea d), which permit unrestricted use, distribution, and reproduction in any medium, provided the original work is properly credited.

\section{REFERENCES}

1. Tanaka Y, Nakayamada S, Okada Y. Osteoblasts and osteoclasts in bone remodeling and inflammation. Curr Drug Targets Inflamm Allergy 2005; 4: 325-328.

2. Burr DB, Forwood MR, Fyhrie DP, Martin RB, Schaffler $\mathrm{MB}$, Turner $\mathrm{CH}$. Bone microdamage and skeletal fragility in osteoporotic and stress fractures. J Bone Miner Res 1997; 12: 6-15.

3. Wade SW, Strader C, Fitzpatrick LA, Anthony MS, O'Malley $C D$. Estimating prevalence of osteoporosis: examples from industrialized countries. Arch Osteoporos 2014; 9: 1-10.

4. Schnatz PF, Marakovits KA, O'Sullivan DM. Assessment of postmenopausal women and significant risk factors for osteoporosis. Obstet Gynecol Surv 2010; 65: 591596.

5. He L, Hannon GJ. MicroRNAs: small RNAs with a big role in gene regulation. Nat Rev Genet 2004; 5: 522 531.

6. E Nicolas F, F Lopez-Martinez A. MicroRNAs in human diseases. Recent Pat DNA Gene Seq 2010; 4: 142-154.

7. Sugatani T, Hruska KA. MicroRNA-223 is a key factor in osteoclast differentiation. J Cell Biochem 2007; 101: 996-999.

8. Li H, Xie H, Liu W, Hu R, Huang B, Tan Y-F, Liao E-Y, Xu $K$, Sheng Z-F, Zhou $H-D$. A novel microRNA targeting HDAC5 regulates osteoblast differentiation in mice and contributes to primary osteoporosis in humans. The $J$ Clin Invest 2009; 119: 3666-3677.

9. Xiao WZ, Gu XC, Hu B, Liu XW, Zi Y, Li M. Role of microRNA-129-5p in osteoblast differentiation from bone marrow mesenchymal stem cells. Cell Mol Biol (Noisyle-grand) 2015; 62: 95-99.

10. Chen $X$, Ba Y, Ma L, Cai X, Yin Y, Wang K, Guo J, Zhang $Y$, Chen J, Guo X. Characterization of microRNAs in serum: a novel class of biomarkers for diagnosis of cancer and other diseases. Cell Res 2008; 18: $997-$ 1006.
11. Meng J, Zhang D, Pan N, Sun N, Wang Q, Fan J, Zhou $P$, Zhu W, Jiang L. Identification of miR-194-5p as a potential biomarker for postmenopausal osteoporosis. Peer J 2015; 3: e971.

12. Wang $Y$, Li L, Moore BT, Peng $X-H$, Fang $X$, Lappe JM, Recker RR, Xiao P. MiR-133a in human circulating monocytes: a potential biomarker associated with postmenopausal osteoporosis. PLoS One 2012; 7: e34641.

13. Cao Z, Moore BT, Wang Y, Peng X-H, Lappe JM, Recker $R R$, Xiao P. MiR-422a as a potential cellular microRNA biomarker for postmenopausal osteoporosis. PLoS One 2014; 9: e97098.

14. Handa R, Kalla AA, Maalouf G. Osteoporosis in developing countries. Best Pract Res Clin Rheumatol 2008; 22: 693-708.

15. Seeliger C, Karpinski K, Haug AT, Vester H, Schmitt A, Bauer JS, van Griensven M. Five freely circulating miRNAs and bone tissue miRNAs are associated with osteoporotic fractures. J Bone Miner Res 2014; 29: 1718-1728.

16. Tan L, Yu J-T, Liu Q-Y, Tan M-S, Zhang W, Hu N, Wang $Y$ - L, Sun $L$, Jiang $T$, Tan $L$. Circulating miR-125b as a biomarker of Alzheimer's disease. J Neurol Sci 2014; 336: 52-56.

17. D'Angelo E, Fassan M, Maretto I, Pucciarelli S, Zanon C, Digito M, Rugge M, Nitti D, Agostini M. Serum miR-125b is a non-invasive predictive biomarker of the preoperative chemoradiotherapy responsiveness in patients with rectal adenocarcinoma. Oncotarget 2016; 7: 28647 28657.

18. Nie CL, Ren WH, Ma Y, Xi JS, Han B. Circulating miR$125 \mathrm{~b}$ as a biomarker of Ewing's sarcoma in Chinese children. Genet Mol Res 2015; 14: 19049-19056.

19. Heneghan HM, Miller N, Lowery AJ, Sweeney KJ, Newell $J$, Kerin MJ. Circulating microRNAs as novel minimally invasive biomarkers for breast cancer. Ann Surg 2010; 251: 499-505.

20. Yuxia M, Zhennan T, Wei Z. Circulating miR-125b is a novel biomarker for screening non-small-cell lung cancer and predicts poor prognosis. J Cancer Res Clin Oncol 2012; 138: 2045-2050.

21. World Medical Association. World Medical Association Declaration of Helsinki. Ethical principles for medical research involving human subjects. Bull World Health Organ 2001; 79(4): 373. 